\title{
A Characterization of Solutions of Quadratic BSDEs and a New Approach to Existence*
}

\author{
Joe Jackson $^{\dagger} \quad$ Gordan Žitković ${ }^{\ddagger}$
}

January 26, 2022

\begin{abstract}
We provide a novel characterization of the solutions of a quadratic BSDE, which is analogous to the characterization of local martingales by convex functions. We then use our main result to show that BSDE solutions are closed under ucp convergence. Finally, we use our closure result obtain a sufficient condition for existence, and discuss specific cases in which this sufficient condition can be verified.
\end{abstract}

\section{Introduction}

Backward Stochastic Differential Equations (BSDEs) were first introduced by Bismut [Bis73] in 1973 in the linear case, and later generalized by Pardoux and Peng [PP90], who studied them under general Lipschitz conditions. In this paper we are concerned with quadratic BSDEs, i.e., BSDEs of the form

$$
d Y=f(t, Y, Z) d t+Z d B,
$$

${ }^{*}$ During the preparation of this work the second author has been supported by the National Science Foundation under Grant No. DSM1815017 (2018-2021). Any opinions, findings and conclusions or recommendations expressed in this material are those of the author(s) and do not necessarily reflect the views of the National Science Foundation (NSF).

†Department of Mathematics, The University of Texas at Austin, Austin, TX jjackso1@utexas.edu

${ }^{\ddagger}$ Department of Mathematics, The University of Texas at Austin, Austin, TX gordanz@math . utexas. edu 
with $Y$ taking values in some $\mathbb{R}^{n}$ (systems of BSDEs), $Z$ in $\mathbb{R}^{n \times d}$, whose driver $f$ has quadratic growth in $z$. In 2000, Kobylanski [Kob00] established the well-posedness of such one-dimensional quadratic BSDEs with a bounded terminal condition. For a survey of the most important results in this active field up to 2017, we refer the reader to the textbook [Zha17] of Zhang.

Well-posedness for multi-dimensional quadratic BSDEs has proved more challenging and is still a central open problem. The most general results in this direction come from Tevzadze [Tev08] under a smallness assumption, and from Xing and Žitković [XŽ18] in the Markovian setting. We refer the reader to [HT16, JKL17, CN17, Nam19, HR19] and the references therein for some other important contributions.

The importance of BSDEs in optimal stochastic control and stochastic game theory is well documented (see, e.g., the monographs [Pha09] or [Car16] and the references therein). It is not as well known that quadratic BSDEs also appear in stochastic differential geometry, as pioneered by Darling in [Dar95], based on the earlier work of Kendall, Picard and others (see, e.g., [Ken90, Ken94, Ken91, Ken92, Pic94] and the references therein). Let $\Gamma$ be an affine connection on a differentiable manifold $M$ which, for the sake of simplicity, we take to be diffeomorphic to $\mathbb{R}^{n}$. Martingales on $M$ (also called $\Gamma$-martingales) are manifold-valued processes that generalize continuous local martingales and share many of their properties (see, e.g., [Éme89, Chapter IV] for an overview). One such property is the following: an $M$-valued process $Y$ is a $\Gamma$-martingale if and only if $\phi(Y)$ is a submartingale whenever $\phi$ is a (locally defined) convex function (see [Éme89, Theorem 4.9, p. 43] for a precise statement). As in the Euclidean case, this characterization gives an analytic insight into various properties of martingales, and can be used to prove, among others, the fundamental result that the set of $\Gamma$-martingales is closed under the ucp (uniform on compacts in probability) convergence (see [Éme89, Theorem 4.43, p. 47]).

The relationship between $\Gamma$-martingales and BSDEs stems from the simple observation that a process $Y$, adapted with respect to a Brownian filtration and taking values in $M$, is a $\Gamma$-martingale if and only if it solves the BSDE

$$
d Y=f(Y, Z) d t+Z d B \text { with } f^{i}(y, z)=\frac{1}{2} \sum_{i_{1}, i_{2}=1}^{n} \sum_{j=1}^{d} \Gamma_{i_{1}, i_{2}}^{i}(y) z_{j}^{i_{1}} z_{j}^{i_{2}},
$$

where $n$ is the dimension of the manifold, $d$ is the dimension of the driving Brownian motion, and $\Gamma_{i_{1}, i_{2}}^{i}$ are the Christoffel symbols of the affine connection on $M$. 
Using this observation, Darling constructed $\Gamma$-martingales with prescribed terminal conditions and studied their properties. In his analysis, he used the fact that the family of $\Gamma$-martingales is closed under ucp convergence as a key tool in addressing the well-posedness of BSDEs of the special form (1.2) under geometric restrictions on the Christoffel symbols $\Gamma$. We note that the equation (1.2) can be viewed as a BSDE on a manifold, written in local coordinates. This perspective is developed in [Bla05] and [Bla06], where more general BSDEs on manifolds are treated, and the results of [Dar95] for the equation (1.2) are extended considerably.

\subsection{Our results}

Inspired by the connection between special BSDEs and $\Gamma$-martingales considered by Darling, we set out to investigate the following questions: In which ways do solutions to general BSDEs behave like martingales? Just as Darling used a special class of BSDEs to describe martingales on manifolds, can we tie a properly generalized notion of a martingale to a general BSDE? Is there a geometric picture associated with such a generalization?

Being the starting point in this program, the present paper answers a more specific question: Is there a "convex-function" characterization of solutions to BSDEs in the spirit of Emery? Indeed, since martingales are characterized by the way they transform under convex maps, can we do the same with solutions of BSDEs? Of course, classical convex functions - which turn each martingale into a local submartingale - will not work for a BSDE with a general driver $f$. We show that the suitable notion is that of $f$-subharmonicity and, given an Itô-process $Y$, we give the following characterization: there exists a process $Z$ such that $(Y, Z)$ solves (1.1) if and only if $\phi(t, B, Y)$ is a submartingale whenever $\phi$ is an $f$-subharmonic function. In the case $f \equiv 0, f$-subharmonic functions coincide with $C^{1,2,2}$-convex functions, while in the "geometric" case of Darling, they coincide with geodesically convex functions on the manifold. The general case is considerably more complicated than either of the two special cases mentioned above and corresponds to the class of sufficiently smooth subsolutions to a fully nonlinear parabolic PDE. We note that our notion of $f$-subharmonicity resembles the notion of a Lyapunov function introduced in [XŽ18], but it is not strictly comparable to it and its use is quite different. We note also that Proposition 2.4.1 of [Bla05] is similar in spirit to our Theorem 2.5, although in a much more restrictive geometric setting, where Emery's proof that martingales on manifolds are characterized by convex functions is easier to adapt. 
The supermartingale property under an exponential transformation in dimension $n=1$ has been used by [BE13a] to characterize certain classes of semimartingales and prove powerful closure results under monotone convergence. These results also lead to an alternative proof of a part of Kobylanski's theorem.

One of the most important features of $f$-subharmonic functions is that they naturally come defined only locally, and that local $f$-subharmonic functions cannot always be extended to a larger (full) domain. This property is well known in the case of geodesically-convex functions on certain Riemannian manifolds, even those diffeomorphic to a Euclidean space. Interestingly enough, this feature kicks in only in dimensions higher than 1, and only with drivers exhibiting a truly quadratic growth in $z$. It also explains why our characterization needs to be local in nature - sometimes, quite simply, there are no non-trivial globally defined $f$-subharmonic functions.

Dimensionality also plays a role when it comes to the most basic features of processes that become submartingales under $f$-subharmonic transformations. In dimension 1, such processes are automatically Itô-processes, but in higher dimensions additional structure is needed to rule out the presence of singular drift components (see Corollary 2.12).

After proving Theorem 2.5, we use our characterization to prove that, under appropriate conditions, the set of processes $Y$ which solve (1.1) (for some terminal condition) is closed under ucp limits. We note that this closure result holds in a high degree of generality when $n=1$, but some additional structure is needed when $n>1$, see Remark 2.10. This result, namely Theorem 3.1, is directly analogous to the parallel result about $\Gamma$-martingales on manifolds mentioned above.

Finally, we present in sub-section 3.2 a "template for existence", i.e. a sufficient condition for the existence of solutions to quadratic BSDE systems. This result, namely Proposition 3.5, shows how the geometric arguments in [Dar95] might eventually be applied to general quadratic systems, and its proof is based on the ucp closure result Theorem 3.1. We note that it is possible to verify the hypotheses of Proposition 3.5 in certain special cases, see Remark 3.7.

\subsection{Organization of the Paper.}

In Section 2, we describe notation and assumptions, introduce the notion of $f$ subharmonic functions and $f$-local martingales, and then state and prove the main result of the paper. In Section 3 we show ucp-stability solutions of BSDEs, and then 
present a template for existence.

\subsection{Notation and conventions.}

Throughout the paper, we work on a fixed probability space $(\Omega, \mathbb{F}, \mathbb{P})$ which hosts a $d$-dimensional Brownian motion $B$ with a deterministic time horizon $T<\infty$. The filtration $\mathbb{F}=\left(\mathcal{F}_{t}\right)_{t \in[0, T]}$ is the augmented filtration of $B$, while the augmented filtration generated by a process $X$ is denoted by $\mathbb{F}^{X}$.

Components of vectors in $\mathbb{R}^{n}$ get indices based on $i$ (such as $i$ or $i^{\prime}$ ), written as superscripts. For $\mathbb{R}^{d}$-valued vectors, indices are based on $j$ and are written as subscripts. Vectors in $\mathbb{R}^{n \times d}$ are interpreted as $n \times d$-matrices, and we use indexing of the form $z=\left(z_{j}^{i}\right)$, with the understanding that $z^{i}$ denotes the $i$-th row and $z_{j}$ $j$-th column. Indices based on $i$ always range from 1 to $n$, and those based on $j$ from 1 to $d$. This way, for example, $\sum_{i}=\sum_{i=1}^{n}$ and $\sum_{i^{\prime}, j}=\sum_{i^{\prime}=1}^{n} \sum_{j=1}^{d}$. Finally, we do not use the Einstein summation convention.

The standard inner product $\langle\cdot, \cdot\rangle$ is used on $\mathbb{R}^{n}$ and $\mathbb{R}^{d}$. In the matrix (i.e., $\mathbb{R}^{n \times d}$ ) case, we use the Frobenius inner product given by $\left\langle z, z^{\prime}\right\rangle=\sum_{i, j} z_{j}^{i} z_{j}^{\prime i}=\operatorname{Tr} z^{T} z^{\prime}$. In all three cases, we use the induced norm, denoted by $|\cdot|$.

$\mathcal{P}$ denotes the set of all $\mathbb{F}$-progressively measurable $\mathbb{R}^{n \times d}$-valued processes, while $\mathcal{P}^{p}$ is the set of all $Z \in \mathcal{P}$ such that $\int_{0}^{T}\left|Z_{t}\right|^{p} d t<\infty$, a.s. For $Z \in \mathcal{P}^{2}, \int Z \cdot d B$ is $\mathbb{R}^{n}$-valued process defined by $\left(\int Z \cdot d B\right)^{i}:=\sum_{j} \int Z_{j}^{i} d B_{j} \cdot \mathcal{S}^{p}$ denotes the set of all continuous adapted $\mathbb{R}^{n}$-valued processes $Y$ with $\|Y\|_{\mathcal{S}^{p}}:=\left\|Y_{T}^{*}\right\|_{\mathbb{L}^{p}}<\infty$. bmo denotes the set of all processes $Z \in \mathcal{P}^{2}$ such that $\int Z \cdot d B$ is a BMO martingale, and $\|Z\|_{\mathrm{bmo}}:=\left\|\int Z \cdot B\right\|_{\mathrm{BMO}}$, where $\|\cdot\|_{\mathrm{BMO}}$ denotes the standard BMO norm.

\section{A characterization of solutions}

This section gives a novel characterization the class of processes $Y$ which solve a given BSDE. A central role in this characterization will be played by the class of $f$-subharmonic functions, introduced below. 


\subsection{Basic assumptions.}

Given a continuous driver $f:[0, T] \times \mathbb{R}^{n} \times \mathbb{R}^{n \times d} \rightarrow \mathbb{R}^{n}$, a pair of processes $(Y, Z)$ is said to be a solution to

$$
Y_{T}^{i}=Y_{t}^{i}-\int_{t}^{T} f^{i}\left(s, Y_{s}, Z_{s}\right) d s+\int_{t}^{T} Z_{s}^{i} \cdot d B_{s}, 1 \leq i \leq n,
$$

if $Y^{i}$ is a continuous $n$-dimensional $\mathbb{F}$-semimartingale, $Z \in \mathcal{P}^{2}$ and (2.1) holds for all $1 \leq i \leq n$, and all $t \in[0, T]$, a.s. Note that our solution concept does not come with an a-priori terminal condition or any regularity imposed on $Y$ or $Z$ beyond the bare minimum. In particular, $\int Z \cdot d B$ is not necessarily a (true) martingale.

In this paper we will abuse terminology and say that $Y$ (alone) is a solution to (2.1) if there exists $Z \in \mathcal{P}^{2}$ such that $(Y, Z)$ is a solution in the sense described above.

The following assumption can be viewed as a quantitative version of the requirement that $z \mapsto f(t, y, z)$ has quadratic growth for each $t$ and $y$.

Assumption 2.1. The driver $f:[0, T] \times \mathbb{R}^{n} \times \mathbb{R}^{n \times d} \rightarrow \mathbb{R}^{n}$ is jointly continuous and there exists a continuous increasing function $\kappa:[0, \infty) \rightarrow[0, \infty)$ such that for all $(t, y) \in[0, T] \times \mathbb{R}^{n}$ and $z, z^{\prime} \in \mathbb{R}^{n \times d}$, we have

$$
\left|f\left(t, y, z^{\prime}\right)-f(t, y, z)\right| \leq \kappa(|y|)\left(1+|z|+\left|z^{\prime}\right|\right)\left|z^{\prime}-z\right|
$$

\subsection{The notion of $f$-subharmonicity.}

When $Y$ is a solution to (2.1) and $\phi:[0, T] \times \mathbb{R}^{d} \times \mathbb{R}^{n} \rightarrow \mathbb{R}$ is a $C^{1,2,2}$-function, Itô's formula implies that

$$
d \phi\left(t, B_{t}, Y_{t}\right)=\mathcal{L}^{f} \phi\left(t, B_{t}, Y_{t} ; Z_{t}\right) d t+d L_{t}
$$

where $L$ is a local martingale and $\mathcal{L}^{f} \phi:[0, T] \times \mathbb{R}^{d} \times \mathbb{R}^{n} \times \mathbb{R}^{n \times d} \rightarrow \mathbb{R}$, is defined by

$$
\begin{aligned}
\mathcal{L}^{f} \phi(t, x, y ; z) & =-\sum_{i} \phi_{y^{i}}(t, x, y) f^{i}(t, y, z) \\
& +\phi_{t}(t, x, z)+\frac{1}{2} \sum_{i, i^{\prime}} \phi_{y^{i} y^{i^{i}}}(t, x, y)\left\langle z^{i}, z^{i^{\prime}}\right\rangle \\
& +\sum_{i, j} \phi_{x_{j} y^{i}}(t, x, y) z_{j}^{i}+\frac{1}{2} \sum_{j} \phi_{x_{j} x_{j}}(t, x, y) .
\end{aligned}
$$

Therefore, if $\phi$ is a $C^{1,2,2}$-function with the property that $\mathcal{L}^{f} \phi \geq 0$ everywhere, the composition $\phi\left(t, B_{t}, Y_{t}\right)$ is necessarily a local submartingale. A local version of this property motivates the the following definition: 
Definition 2.2 ( $f$-subharmonic functions). A real valued $C^{1,2,2}$-function $\phi$ defined on an open set $\operatorname{dom} \phi$ of $[0, T] \times \mathbb{R}^{d} \times \mathbb{R}^{n}$ is said to be $f$-subharmonic if

$$
\inf _{z \in \mathbb{R}^{n \times d}} \mathcal{L}^{f} \phi(t, x, y ; z) \geq 0 \text { for all }(t, x, y) \in \operatorname{dom} \phi .
$$

The set of all $f$-subharmonic functions is denoted by $S^{f}$.

Remark 2.3. Probabilistically, one can interpret $f$-subharmonicity as a sufficient condition for a function to transform any solution $Y$ of (2.1) to a submartingale "while $\left(t, B_{t}, Y_{t}\right) \in \operatorname{dom} \phi$ ". To understand $f$-subharmonicity better from the analytic point of view, we consider only the case where $\phi$ does not depend on $t$ and start in the simplest setting with $f=0$ and $n=d=1$ in which

$$
\mathcal{L}^{0} \phi(t, x, y ; z)=\frac{1}{2}\left\langle\zeta, D^{2} \phi(t, x, y) \zeta\right\rangle \text { where } \zeta=(1, z) \text { and }
$$

$D^{2} \phi(t, x, y)$ is the Hessian matrix of $\phi$ in variables $x$ and $y$. It follows immediately that $\phi$ is $f$-subharmonic if and only if $D^{2} \phi$ is non-negative definite on dom $\phi$, which is, in turn, equivalent to joint convexity of $\phi$ in $x$ and $y$ on $\operatorname{dom} \phi$.

When $n=1$ but $d>1, f$-subharmonicity, even for $f=0$, no longer implies convexity. Indeed, in that case we have

$$
\mathcal{L}^{0} \phi(t, x, y ; z)=\frac{1}{2}\langle\xi, H \xi\rangle,
$$

where $\xi=\left(|z|, z_{1} /|z|, \ldots, z_{n} /|z|\right)$ (with $z_{i} /|z|:=0$ when $|z|=0$ ) and the matrix $H$ is given by

$$
H=\left(\begin{array}{ccccc}
\phi_{y y} & \phi_{y x_{1}} & \phi_{y x_{2}} & \ldots & \phi_{y x_{d}} \\
\phi_{x_{1} y} & \Delta_{x} \phi & 0 & \ldots & 0 \\
\phi_{x_{2} y} & 0 & \Delta_{x} \phi & \ldots & 0 \\
\vdots & \vdots & \vdots & \ddots & \vdots \\
\phi_{x_{d} y} & 0 & 0 & \ldots & \Delta_{x} \phi
\end{array}\right) .
$$

As above, $f$-subharmonicity is equivalent to non-negative definiteness of the matrix $H$, but $H$ is no longer the Hessian of $\phi$. It is, however, obtained from the Hessian $D^{2} \phi$ by replacing the submatrix of $\left(x_{j}, x_{j^{\prime}}\right)$-derivatives by a $\Delta_{x} \phi$-multiple of the $n \times n$ identity matrix. When $\phi$ does not depend on $y, 0$-subharmonicity is equivalent to classical subharmonicity of $\phi$, a property strictly weaker than convexity. In general, the notion of 0-subharmonicity can be interpreted as a "convex combination" of convexity and subharmonicity. 
The general case, when $f$ does not necessarily vanish, is much harder to interpret, primarily because the fact that the variable $z$, which we used to test positive definiteness above no longer separates from the rest. One special case where such a separation does occur is when $f(t, x, y, \cdot)$ is a quadratic form in $z$, i.e.,

$$
f(t, x, y, z)=\sum_{i, i^{\prime}} F_{i, i^{\prime}}(t, x, y) z^{i} z^{i^{\prime}}
$$

a particular case of which we have seen in (1.2) in the Introduction. We hope it may shed some light on a possible deeper geometric meaning of $f$-subharmonicity; it also leads to non-negative definiteness, but of a matrix $H^{\prime}$ which is obtained from $H$ above by adding certain first-order terms to it, just as in the the coordinate representation of the covariant Hessian on a differentiable manifold with an affine connection.

\subsection{The main result}

With the family $S^{f}$ of all $f$-subharmonic functions introduced in Definition 2.2 above, we give two more definitions whose primary purpose is to improve presentation of our main theorem and its proof. Given a stochastic process $\Phi$ and a stopping time $\tau$, we denote by $\Phi^{\tau}$ the process $\Phi$ stopped at time $\tau$, i.e. $\Phi_{t}^{\tau}=\Phi_{\tau \wedge t}$. For two stopping times $\tau_{1} \leq \tau_{2}$ and a stochastic process $\Phi$, we define

${ }^{\tau_{1}} \Phi_{t}^{\tau_{2}}:=\Phi_{t \vee \tau_{1}}^{\tau_{2}}-\Phi_{\tau_{1}}^{\tau_{2}}=\left(\Phi_{t}-\Phi_{\tau_{1}}\right) 1_{\left[\tau_{1}, \tau_{2}\right)}(t)+\left(\Phi_{\tau_{2}}-\Phi_{\tau_{1}}\right) 1_{\left[\tau_{2}, T\right]}(t)=\int_{0}^{t} 1_{\left(\tau_{1}, \tau_{2}\right]}(u) d \Phi_{u}$.

We say that $Y$ is a local submartingale on $\left[\tau_{1}, \tau_{2}\right)$ if ${ }^{\tau_{2}} Y^{\tau_{1}}$ is a local submartingale.

Definition 2.4 (Local $f$-martingales).

1. Given a function $\phi$ defined on an open subset $U$ of $[0, T] \times \mathbb{R}^{m}$, and an $\mathbb{R}^{m}$ valued continuous adapted process $X$, we say that $Y_{t}=\phi\left(t, X_{t}\right)$ is a local submartingale while $(t, X) \in U$ if it is a local submartingale on each stochastic interval $\left[\tau_{1}, \tau_{2}\right)$ such that $\left(t, X_{t}\right) \in U$ for all $t \in\left[\tau_{1}, \tau_{2}\right)$, a.s.

2. A continuous adapted process $Y$ is called a local $f$-martingale if $\phi\left(t, B_{t}, Y_{t}\right)$ is a local submartingale while $(t, B, Y) \in \operatorname{dom} \phi$ for each $\phi \in S^{f}$.

The main result of the paper is the following characterization: 
Theorem 2.5 (Main). Under Assumption 2.1, an Itô process $Y$ is a local $f$ martingale if and only if it solves the BSDE (2.1).

With the proof left for the following section, here are some comments on the form, scope and conditions of Theorem 2.5.

Remark 2.6.

1. As we show in Corollary 2.12 below, the a-priori assumption that $Y$ is an Itô process is not necessary if either $n=1$ or $f$ satisfies an additional structural condition; a local $f$-martingale is automatically a semimartingale and an Itôprocess in that case. An inspection of our proof of Theorem 2.5 below reveals that when $n>1$, local $f$-martingales correspond to possibly generalized solutions of (2.1) where a singular finite-variation process $A^{i}$ is added to each equation and treated as component of the solution.

2. Theorem 2.5 would remain valid if we replaced the Brownian motion $B$ in the definition of $f$-subharmonic function by a Markov process in an appropriate class. Of course, the definition of an $f$-subharmonic function would have to be suitably modified. We leave the problem of identifying the range of possible replacements for $B$ for further research.

Clearly, full $\omega$-dependence cannot be expected even under strong adaptivity assumptions without changing the nature of our result in a fundamental way. Indeed, it would require replacing the notion of an $f$-subharmonic function by a random field, and, as such, could not be used to separate analytic and probabilistic aspects of BSDEs.

3. The notion of a solution of a BSDE at our level of generality can be thought of as a particular form of dependence between a process $Y$ and the given Brownian motion. Therefore, the variable $B$, or some surrogate, cannot be left out completely out of the definition of an $f$-subharmonic function. Indeed, consider the case $n=1, f(t, y, z)=z$, and the class $S \subseteq S^{f}$ consisting of all $\phi$ in $S^{f}$ that do not depend on $x$. Whether or not a process $\phi\left(t, Y_{t}\right)$ is a local submartingale for each $\phi \in S$ depends only on the distribution of $Y$, so it will be enough to exhibit two processes equal in distribution, such that one solves (2.1), but the other does not. For example, we may simply take

$$
Y_{t}=t+B_{t} \text { and } Y_{t}^{\prime}=t+\int_{0}^{t} h_{s} d B_{s}
$$


where $h$ is any $\{-1,1\}$-valued progressive process not equal to $1,(\lambda \otimes \mathbb{P})$-a.e. It is clear that both of these are Brownian motions with a unit drift, and that the first one solves (2.1) with $Z=1$. The other one is not a solution since the only candidate for the second component of the solution, namely $Z_{t}^{\prime}=h_{t}$, does not satisfy (2.1).

\subsection{Proof of Theorem 2.5.}

In this subsection, we provide a proof of Theorem 2.5. We then show how the hypotheses Theorem 2.5 can be weakened when $n=1$ or $f$ satisfies an additional structural condition. We fix a driver $f$ which satisfies Assumption 2.1, and start with some auxiliary results.

Given $\bar{z} \in \mathbb{R}^{n \times d}$, we will call a function of the form $g(z)=a_{0}+b_{0}|z-\bar{z}|+c_{0}|z-\bar{z}|^{2}$ with $c_{0} \geq 0$ cone-quadratic about $\bar{z}$, and a function of the form $h(z)=d_{0}+e_{0} \mid z-$ $\left.\bar{z}\right|^{2}$ with $e_{0} \geq 0$ purely quadratic about $\bar{z}$. We identify such functions with points in $\mathbb{R}^{2} \times[0, \infty)$ and $\mathbb{R} \times[0, \infty)$, respectively, defined by their coefficients. This way we can speak about neighborhoods around cone-quadratic or purely quadratic functions in the following, simple, lemma whose proof we leave to the reader:

Lemma 2.7. For any $\varepsilon>0, \bar{z} \in \mathbb{R}^{n \times d}$ and a cone-quadratic function $l$ about $\bar{z}$ there exists a purely quadratic function $q$ about $\bar{z}$ and neighborhoods $Q$ around $q$ and $L$ around $l$ such that

1. $\tilde{l}(z) \leq \tilde{q}(z)$ for all $z \in \mathbb{R}^{n}, \tilde{l} \in L$ and $\tilde{q} \in Q$,

2. $q(\bar{z}) \leq l(\bar{z})+\varepsilon$

Furthermore, $q$ can be chosen non-constant. That is, we can take $q(z)=d_{0}+e_{0} \mid z-$ $\left.z_{0}\right|^{2}$ with $e_{0}>0$.

The next lemma shows that $\phi$-subharmonic functions exist locally, even when their behavior at a given point is additionally restricted.

Lemma 2.8. For any $(\bar{t}, \bar{x}, \bar{y}, \bar{z}) \in[0, T] \times \mathbb{R}^{d} \times \mathbb{R}^{n} \times \mathbb{R}^{n \times d}, 1 \leq i_{0} \leq n$ and $\epsilon>0$, there exists an $f$-subharmonic function $\phi$ such that

1. $(\bar{t}, \bar{x}, \bar{y}) \in \operatorname{dom} \phi$,

2. $\mathcal{L}^{f} \phi(\bar{t}, \bar{x}, \bar{y} ; \bar{z}) \leq \epsilon$ and 
3. $\phi_{y^{i_{0}}}(\bar{t}, \bar{x}, \bar{y})=1$ (or -1 if desired), and

4. $\phi_{y^{i}}(\bar{t}, \bar{x}, \bar{y})=0$ for $i \neq i_{0}$.

Proof. We make the following Ansatz

$$
\phi(t, x, y)=-\sum_{i \neq i_{0}} y^{i}+\sum_{i} \frac{1}{\beta^{i}} E^{i}(x, y)+\theta|x|^{2}
$$

where

$$
E^{i}(x, y)=e^{\beta^{i}\left(y^{i}-\bar{y}^{i}\right)+\left\langle\gamma^{i}, x-\bar{x}\right\rangle},
$$

and the constants $\theta \in \mathbb{R}, \beta \in \mathbb{R}^{n}$ and $\gamma \in \mathbb{R}^{n \times d}$, as well as the domain dom $\phi$ will be determined later. It follows by direct computation that

$$
\begin{aligned}
\phi_{y^{i}}(t, x, y) & =E^{i}(x, y)+\delta^{i i_{0}}-1, & & \phi_{y^{i} y^{i}}(t, x, y)=\beta^{i} E^{i}(x, y), \\
\phi_{x_{j} x_{j}}(t, x, y) & =\sum_{i} \frac{\left(\gamma_{j}^{i}\right)^{2}}{\beta^{i}} E^{i}(x, y)+2 \theta, & & \phi_{y^{i} x_{j}}(t, x, y)=\gamma_{j}^{i} E^{i}(x, y),
\end{aligned}
$$

where $\delta^{*}=1_{.=}$. is the Kronecker delta function, so that

$$
\begin{aligned}
\mathcal{L}^{f} \phi(t, x, y ; z)= & -\sum_{i}\left(E^{i}(x, y)+\delta^{i i_{0}}-1\right) f^{i}(t, y, z)+\frac{1}{2} \sum_{i} \beta^{i} E^{i}(x, y)\left|z^{i}\right|^{2} \\
& +\sum_{i, j} \gamma_{j}^{i} E^{i}(x, y) z_{j}^{i}+\frac{1}{2} \sum_{i, j}\left(\gamma_{j}^{i}\right)^{2} \frac{1}{\beta^{i}} E^{i}(x, y)+\theta d .
\end{aligned}
$$

Next, we define the function $l:[0, T] \times \mathbb{R}^{d} \times \mathbb{R}^{n} \times \mathbb{R}^{n \times d} \rightarrow \mathbb{R}$ by

$$
\begin{aligned}
l(t, x, y, z):= & \sum_{i}\left|E^{i}(x, y)+\delta^{i i_{0}}-1\right|\left(\left|f^{i}(t, y, \bar{z})-f^{i}(\bar{t}, \bar{y}, \bar{z})\right|\right. \\
& +C(\kappa(|y|)+1)\left(|z-\bar{z}|+|z-\bar{z}|^{2}\right)+\sum_{i}\left(E^{i}(x, y)+\delta^{i i_{0}}-1\right) f^{i}(\bar{t}, \bar{y}, \bar{z}),
\end{aligned}
$$

where $C>0$ is a constant such that

$$
f^{i}(t, y, z) \leq f^{i}(\bar{t}, \bar{y}, \bar{z})+\left|f^{i}(t, y, \bar{z})-f^{i}(\bar{t}, \bar{y}, \bar{z})\right|+C \kappa(|y|)\left(|z-\bar{z}|+|z-\bar{z}|^{2}\right)
$$

for all $t, y, z$ and $i$. Such a choice of $C$ - which exists thanks to Assumption 2.1 and repeated application of the triangle inequality - leads to the following tight inequality:

$$
\begin{aligned}
& l(t, x, y, z) \geq \sum_{i}\left(E^{i}(x, y)+\delta^{i i_{0}}-1\right) f^{i}(t, y, z), \text { for all } t, x, y, z, \text { and } \\
& l(\bar{t}, \bar{x}, \bar{y}, \bar{z})=\sum_{i}\left(E^{i}(\bar{x}, \bar{y})+\delta^{i i_{0}}-1\right) f^{i}(\bar{t}, \bar{y}, \bar{z}) .
\end{aligned}
$$


The $(\bar{t}, \bar{x}, \bar{y})$-section of the function $l$ is a cone-quadratic function of $z$ about $\bar{z}$; Lemma 2.7 applied to it yields a purely quadratic function $q(z)=d_{0}+e_{0}|z-\bar{z}|^{2}$ with $e_{0} \neq 0$, a neighborhood $Q$ around it and a neighborhood $L$ around $l(\bar{t}, \bar{x}, \bar{y}, \cdot)$.

We now choose $\beta^{i}, \gamma^{i}$, and $\theta$ so that

$$
\frac{1}{2} \beta^{i}=e_{0}, \quad \gamma_{j}^{i}=-2 e_{0} \bar{z}_{j}^{i}, \text { and } \frac{1}{2} \sum_{i, j}\left(\gamma_{j}^{i}\right)^{2} \frac{1}{\beta^{i}}+\theta d=d_{0}+e_{0}|\bar{z}|^{2} .
$$

With this choice of constants, we have

$$
\frac{1}{2} \sum_{i} \beta^{i} E^{i}(\bar{x}, \bar{y})\left|z^{i}\right|^{2}+\sum_{i, j} \gamma_{j}^{i} E^{i}(\bar{x}, \bar{y}) z_{j}^{i}+\frac{1}{2} \sum_{i, j}\left(\gamma_{j}^{i}\right)^{2} \frac{1}{\beta^{i}} E^{i}(\bar{x}, \bar{y})+\theta d=q(z) .
$$

The coefficients of $l$ are continuous as functions of $(t, x, y)$, so there exists a neighborhood $U$ of $(\bar{t}, \bar{x}, \bar{y})$ such that $l(t, x, y, \cdot) \in L$, for all $(t, x, y) \in U$. Since $E^{i}(\bar{x}, \bar{y})=1$ for each $i$, we can shrink this neighborhood further, if needed, to guarantee that the map

$$
z \mapsto \frac{1}{2} \sum_{i} \beta^{i} E^{i}(x, y)\left|z^{i}\right|^{2}+\sum_{i, j} \gamma_{j}^{i} E^{i}(x, y) z_{j}^{i}+\frac{1}{2} \sum_{i, j}\left(\gamma_{j}^{i}\right)^{2} \frac{1}{\beta^{i}} E^{i}(x, y)+\theta d .
$$

lies in $Q$ as soon as $(t, x, y) \in U$.

If we declare dom $\phi=U$ the first conclusion of Lemma 2.7 implies that $\phi$ is $f$-subharmonic with $(\bar{t}, \bar{x}, \bar{y}) \in \operatorname{dom} \phi$. For 2., it suffices to use the equality in (2.7) and the second conclusion of Lemma 2.7. Conditions 3. and 4. follow from the computation $\phi_{y^{i}}(\bar{t}, \bar{x}, \bar{y})=E^{i}(\bar{x}, \bar{y})+\delta^{i i^{\prime}}-1=\delta^{i i^{\prime}}$. To handle the constraint $\phi_{y^{i_{0}}}(\bar{t}, \bar{y}, \bar{x})=-1$ we use $E^{i_{0}}(x, y)^{-1}$ instead of $E^{i_{0}}(x, y)$ in the definition of $\phi$ and repeat the argument.

Proof of Theorem 2.5. By design, each solution $Y$ to (2.1) is a local $f$-martingale, so we focus on the opposite implication and proceed by contradiction: we assume that $Y$ is a local $f$-martingale which is not a solution to (2.1).

By assumption, $Y$ is an Itô-process, i.e., admits a decomposition of the form

$$
Y_{t}=Y_{0}-\int_{0}^{t} g_{s} d s+\int_{0}^{t} Z_{s} \cdot d B_{s}
$$

for some locally integrable $g$ and $Z \in \mathcal{P}^{2}$. Since $Y$ does not solve (2.1), the processes $\int_{0}^{\cdot} g_{s}^{i_{0}} d s$ and $\int_{0}^{\cdot} f^{i_{0}}\left(s, Y_{s}, Z_{s}\right) d s$ are not indistinguishable for some $i_{0} \in\{1,2, \ldots, n\}$, which means that

$$
(\lambda \otimes \mathbb{P})\left[g^{i_{0}} \neq f^{i_{0}}(\cdot, Y, Z)\right]:=(\lambda \otimes \mathbb{P})\left[(t, \omega): g_{t}^{i_{0}}(\omega) \neq f^{i_{0}}\left(t, Y_{t}(\omega), Z_{t}(\omega)\right)\right]>0
$$


where $\lambda$ denotes the Lebesgue measure on $[0, T]$. Let us assume first that

$$
(\lambda \otimes \mathbb{P})\left[g^{i_{0}}>f^{i_{0}}(\cdot, Y, Z)\right]>0,
$$

and thus

$$
(\lambda \otimes \mathbb{P})\left[g^{i_{0}}>f^{i_{0}}(\cdot, Y, Z)+\delta\right]>0 \text { for some } \delta>0 .
$$

By considering a countable partition, we can find a cube $C_{1} \subseteq[0, T] \times \mathbb{R}^{d} \times \mathbb{R}^{n} \times \mathbb{R}^{n \times d}$ with side length $T$ and a constant $K>0$ such that

$$
(\lambda \otimes \mathbb{P})\left[(\cdot, B, Y, Z) \in C_{1}, g^{i_{0}}>f^{i_{0}}(\cdot, Y, Z)+\delta,|g|<K,|f(\cdot, B, Y, Z)|<K\right]>0 .
$$

Partitioning $C_{1}$ yields a cube $C_{2}$ of side length $\frac{T}{2}$ with the same property, and, iteratively, we can construct a nested sequence of cubes $C_{n}$ whose diameters go to zero and such that

$$
(\lambda \otimes \mathbb{P})\left[(\cdot, B, Y, Z) \in C_{n}, g^{i_{0}}>f^{i_{0}}(\cdot, Y, Z)+\delta,|g|<K,|f(\cdot, B, Y, Z)|<K\right]>0
$$

We choose a point $(\bar{t}, \bar{y}, \bar{x}, \bar{z}) \in \cap_{n} C_{n}$, and note that any neighborhood $\mathcal{V}$ of $(\bar{t}, \bar{y}, \bar{x}, \bar{z})$ contains some $C_{n}$, and hence satisfies

$$
(\lambda \otimes \mathbb{P})\left[(\cdot, B, Y, Z) \in \mathcal{V}, g^{i_{0}}>f^{i_{0}}(\cdot, Y, Z)+\delta,|g|<K,|f(\cdot, B, Y, Z)|<K\right]>0 .
$$

Given the constant $\delta$ and the point $(\bar{t}, \bar{x}, \bar{y}, \bar{z})$ constructed above, Lemma 2.8 guarantees the existence of an $f$-subharmonic function $\phi$ with $(\bar{t}, \bar{x}, \bar{y}) \in \operatorname{dom} \phi$ such that

$$
\phi_{y^{i} 0}(\bar{t}, \bar{x}, \bar{y})=1, \phi_{y^{i}}(\bar{t}, \bar{x}, \bar{y})=0 \text { for } i \neq i_{0} \text {, and } \mathcal{L}^{f} \phi(\bar{t}, \bar{x}, \bar{y} ; \bar{z})<\delta / 16 \text {. }
$$

By shrinking dom $\phi$ to a smaller neighborhood of $(\bar{t}, \bar{x}, \bar{y})$, if necessary, we can assume further that $\phi_{y^{i 0}}>\frac{1}{2}$ on $\operatorname{dom} \phi, \phi_{y^{i}}<\frac{\delta}{16 n K}$ on $\operatorname{dom} \phi$ for $i \neq k$, and we can find a constant $r>0$ such that $\mathcal{L}^{f} \phi \leq \delta / 8$ on $\operatorname{dom} \phi \times B_{r}(\bar{z})$, where $B_{r}(\bar{z})$ is the open ball in $\mathbb{R}^{n \times d}$ of radius $r$ around $\bar{z}$.

Next, we select a neighborhood $V$ of $(\bar{t}, \bar{x}, \bar{y})$ with $\mathrm{Cl} V \subseteq \operatorname{dom} \phi$ so that the set $W=[0, T] \times \mathbb{R}^{d} \times \mathbb{R}^{n} \backslash \mathrm{Cl} V$ satisfies

$$
W \cup \operatorname{dom} \phi=[0, T] \times \mathbb{R}^{d} \times \mathbb{R}^{n} \text { and } W \cap V=\emptyset .
$$

Here we denote by $\mathrm{Cl} V$ the closure of $V$. The process $\left(t, B_{t}, Y_{t}\right)$ is continuous and $\{\operatorname{dom} \phi, W\}$ is an open cover of $[0, T] \times \mathbb{R}^{d} \times \mathbb{R}^{n}$ so there exists a nondecreasing sequence $\left\{T_{k}\right\}_{k \in \mathbb{N}}$ of $[0, T]$-valued stopping times with the following properties (see, e.g., [Éme89, Lemma 3.5, p. 22]) : 
1. $\mathbb{P}\left[T_{k}<T\right] \rightarrow 0$, as $k \rightarrow \infty$

2. the path $\left(t, B_{t}, Y_{t}\right)$ lies entirely in dom $\phi$ or entirely in $W$ on each stochastic interval $\left[T_{k}, T_{k+1}\right)$.

Since $[0, T]=\cup_{k}\left[T_{k}, T_{k+1}\right)$ a.s., equation (2.8) above guarantees the existence of an index $k_{0} \in \mathbb{N}$ such that

$$
\begin{aligned}
(\lambda \otimes \mathbb{P})[\{(t, \omega): t & \left.\in\left[T_{k_{0}}(\omega), T_{k_{0}+1}(\omega)\right)\right\} \cap\left\{(\cdot, B, Y, Z) \in V \times B_{r}(\bar{z}),\right. \\
& \left.\left.g^{i_{0}}>f^{i_{0}}(\cdot, Y, Z)+\delta,|g|<K,|f(\cdot, B, Y, Z)|<K\right\}\right]>0
\end{aligned}
$$

Moreover, because $W \cap V=\emptyset$, the stopping times

$$
\tau_{1}=T_{k_{0}} \text { and } \tau_{2}=T_{k_{0}+1} \wedge \inf \left\{t \geq \tau_{1}:\left(t, B_{t}, Y_{t}\right) \notin \operatorname{dom} \phi\right\}
$$

have the property that $\left(t, B_{t}, Y_{t}\right) \in \operatorname{dom} \phi$ on $\left[\tau_{1}, \tau_{2}\right)$ as well as

$$
(\lambda \otimes \mathbb{P})\left[\left\{(t, \omega): t \in\left[\tau_{1}(\omega), \tau_{2}(\omega)\right)\right\} \cap \mathcal{K}\right]>0
$$

where

$$
\begin{aligned}
\mathcal{K}=\left\{(\cdot, B, Y, Z) \in V \times B_{r}(\bar{z}),: g^{i_{0}}>f^{i_{0}}(\cdot, Y, Z)+\delta,|g|<K,\right. & |f(\cdot, B, Y, Z)|<K\} .
\end{aligned}
$$

The drift of the process $\phi\left(t, B_{t}, Y_{t}\right)$ can be written as

$$
\begin{aligned}
\mu_{t}=\mathcal{L}^{f} \phi\left(t, B_{t}, Y_{t} ; Z_{t}\right)+\phi_{y^{i_{0}}}\left(t, B_{t}, Y_{t}\right)\left(f^{i_{0}}\right. & \left.\left(t, B_{t}, Y_{t}\right)-g_{t}^{i_{0}}\right)+ \\
& +\sum_{i \neq i_{0}} \phi_{y^{i}}\left(t, B_{t}, Y_{t}\right)\left(f^{i}\left(t, B_{t}, Y_{t}\right)-g_{t}^{i}\right)
\end{aligned}
$$

and using the bounds on its terms enforced by the construction of the domain dom $\phi$ above, we find that

$$
\mu_{t} \leq \delta / 8-\delta / 2+\delta / 8=-\delta / 4,(\lambda \otimes \mathbb{P}) \text {-a.e. on } \mathcal{K} .
$$

On the other hand, $Y$ is a local $f$-martingale so

$$
\mu_{t} \geq 0,(\lambda \otimes \mathbb{P}) \text {-a.e. on }\left[\tau_{1}, \tau_{2}\right),
$$

which, thanks to (2.10), contradicts (2.12).

To deal with the case $(\lambda \otimes \mathbb{P})[g<f(\cdot, Y, Z)]>0$, we use essentially the same argument, but with $\phi_{y^{i 0}}(\bar{t}, \bar{x}, \bar{y})=-1$. 
In anticipation of the closure result Theorem 3.1 below, we wish to identify conditions under which the assumption $Y$ is an Itô process can be removed in the statement of Theorem 2.5. In general, it seems to difficult to rule out the possibility that a local $f$-martingale has singular drift when $n>1$. But, when the driver $f$ has some additional structure, we can indeed guarantee that local $f$-martingales are Itô processes. One structure which works is (a local version of) the condition (AB), introduced in [XŽ18]. We recall the definition here, adapted slightly to meet our needs.

Definition 2.9. A driver $f$ is said to satisfy the condition $(\boldsymbol{A B})$ there exists a finite collection $\left\{a_{m}\right\}=\left(a_{1}, \ldots, a_{M}\right)$ of vectors in $\mathbb{R}^{n}$ such that

1. $a_{1}, \ldots, a_{M}$ positively span $\mathbb{R}^{n}$

2. $a_{m}^{T} f(t, y, z) \leq k+\frac{1}{2}\left|a_{m}^{T} z\right|^{2}$ for each $m$, for all $t, y, z \in[0, T] \times \mathbb{R}^{n} \times \mathbb{R}^{n \times d}$.

We say that $f$ satisfies the condition $(\boldsymbol{A B})$ locally if for each $k \in \mathbb{N}$, the driver $f^{k}(t, y, z):=f\left(t, \pi_{k}(y), z\right)$ satisfies the condition $(A B)$, where $\pi_{k}$ is the orthogonal projection onto the ball of radius $k$ in $\mathbb{R}^{n}$.

Remark 2.10. When $n=1$, Assumption 2.1 already implies that $f$ satisfies the condition (AB) locally. Thus when $n=1$, the hypothesis that $f$ satisfies the condition $(\mathrm{AB})$ locally can be omitted in the statements of the following Lemma and Corollary;.

Lemma 2.11. Suppose that $f$ satisfies the condition $(A B)$ locally. Then each local $f$-martingale is automatically an Itô process.

Proof. By a localization argument, we may assume that $f$ satisfies the condition (AB). Let $k,\left\{a_{m}\right\}$ be as given in the definition of the condition (AB). For each $m$, define $\phi:[0, T] \times \mathbb{R}^{n} \rightarrow \mathbb{R}$ by

$$
\phi_{m}(t, x, y)=\phi_{m}(t, y)=\exp \left(2 a_{m}^{T} y+2 k t\right) .
$$

A computation shows that $\mathcal{L}^{f} \phi_{m}(t, x, y, z)=\exp \left(2 a_{m}^{T} y+2 k t\right)\left(-2 a_{m}^{T} f(t, y, z)+2 k+\right.$ $\left.2\left|a_{m}^{T} z\right|^{2}\right)$. Since $a_{m}^{T} f(t, y, z) \leq k+\left|a_{m}^{T} z\right|^{2}$, we see that

$$
-2 a_{m}^{T} f(t, y, z)+2 k+2\left|a_{m}^{T} z\right|^{2} \geq 0,
$$

and so $\phi_{m}$ is an $f$-subharmonic function for each $m$. Since $Y$ is a local $f$-martingale by hypothesis, it follows that $\phi_{m}(t, Y)=\exp \left(2 a_{m}^{T} Y+2 k t\right)$ is a local submartingale 
for each $m$, hence a semimartingale. The map $(t, y) \mapsto(t, \exp (2 y+2 k t))$ is invertible with $C^{2}$ inverse, so we conclude that $a_{m}^{T} Y$ is a semimartingale for each $m$. Since $\left\{a_{m}\right\}$ spans $\mathbb{R}^{n}$, this shows that $Y$ is a semimartingale. Now that we know $Y$ is a semimartingale, we represent as $Y=Y_{0}-A_{t}+\int_{0}^{t} Z_{s} \cdot d B_{s}$, for a constant $Y_{0}$, continuous finite-variation process $A$, and $Z \in \mathcal{P}^{2}$. The finite-variation part of the process $\phi_{m}(t, Y)$ is given by $D-E$, where

$$
D=2 \int_{0} \exp \left(2 a_{m}^{T} Y+k t\right)\left(\left|a_{m}^{T} Z\right|^{2}+k\right) d t, E=-2 \int_{0} \exp \left(2 a_{m}^{T} Y+k t\right) d\left(a_{m}^{T} A\right) .
$$

Since $\phi_{m}(t, Y)$ is a submartingale, we see that $E \preceq D$ a.s. Thus we can find for each $m$ an absolutely continuous process $H^{m}$ such that $a_{m}^{T} A \preceq H^{m}$. Since $\left\{a_{m}\right\}$ positively spans $\mathbb{R}^{n}$, for each basis vector $e_{i}$ of $\mathbb{R}^{n}$, we may find non-negative scalars $\lambda_{1}, \ldots, \lambda_{M}$ such that $e_{i}=\sum_{m} \lambda_{m} a_{m}$, and so

$$
A^{i}=\sum_{m} \lambda_{m} a_{m}^{T} A^{i} \preceq \sum_{m} \lambda_{m} H^{m} .
$$

But the same argument works for $-e_{i}$, i.e. we may find positive constants $\lambda_{m}^{\prime}$ such that $-e_{i}=\sum_{m} \lambda_{m}^{\prime}$, which leads to

$$
-A^{i}=\sum_{m} \lambda_{m}\left(a_{m}^{\prime}\right)^{T} A^{i} \preceq \sum_{m} \lambda_{m}^{\prime} H^{m} .
$$

We conclude that

$$
-\sum_{m} \lambda_{m}^{\prime} H^{m} \preceq A^{i} \preceq \sum_{m} \lambda_{m} H^{m},
$$

a.s., and so for each $i, A^{i}$ has absolutely continuous paths, a.s. The argument is completed by applying standard theory to produce a progressive density for $A$ (see, e.g., [JS03, Proposition 3.13, p. 30]).

Thanks to Theorem 2.5, we get the following Corollary.

Corollary 2.12. If $f$ satisfies the condition $(A B)$ locally and Assumption 2.1 holds, then a process $Y$ is a local $f$-martingale if and only if it solves (2.1).

Remark 2.13. We note that other structural conditions can be used to guarantee that local $f$-martingales are automatically Itô processes. For example, if $f$ is triangular in the sense of Definition 2.10 of [XŽ18], then an argument very similar to the one appearing in the proof of Lemma 2.11 shows that any local $f$-martingale is an Itô process. 


\section{Applications}

\subsection{Stability of Solutions to BSDE}

The set of solutions to (2.1) shares several properties with the set of continuous local martingales. We focus here on one which concerns stability under ucp convergence and show how it follows from Lemma 2.12. We remind the reader that a sequence $\left\{Y^{k}\right\}_{n \in \mathbb{N}}$ of continuous processes converges uniformly on compacts in probability (in $u c p)$, denoted by $Y^{k} \stackrel{\text { ucp }}{\rightarrow} Y$, if

$$
\sup _{t \in[0, T]}\left|Y_{t}^{k}-Y_{t}\right| \rightarrow 0 \text { in probability. }
$$

Theorem 3.1. Suppose that Assumption 3.2 holds, and that $f$ satisfies the condition (AB) locally. Then the set of solutions to (2.1) is closed under ucp convergence.

Proof. Let $\left\{Y_{k}\right\}_{k \in \mathbb{N}}$ be a sequence of solutions of (2.1) such that $Y^{k} \stackrel{\text { ucp }}{\rightarrow} Y$. Clearly the limit process $Y$ is continuous and adapted. To show that it solves (2.1) via Corollary 2.12, we pick $\phi \in S^{f}$ and a pair of stopping times $\tau_{1}, \tau_{2}$ such that $\left(t, Y_{t}, B_{t}\right) \in \operatorname{dom} \phi$ for $t \in\left[\tau_{1}, \tau_{2}\right)$, a.s. In fact, it is clear from the proofs of Theorem 2.5 and Lemma 2.11 we need only consider $\phi$ such that there exists $\tilde{\phi} \in S^{f}$ with $\mathrm{Cl} \operatorname{dom} \phi \subset \operatorname{dom} \tilde{\phi}, \phi=\tilde{\phi}$ on $\operatorname{dom} \phi$, and $\phi$ is Lipschitz on dom $\phi$.

For $k \in \mathbb{N}$, let the stopping time $S_{k}$ be defined by

$$
S_{k}:=\tau_{2} \wedge \inf \left\{t \geq \tau_{1}:\left(t, B_{t}, Y_{t}^{k}\right) \notin \operatorname{dom} \tilde{\phi}\right\}
$$

so that $\left(t, B, Y_{t}^{k}\right) \in \operatorname{dom} \tilde{\phi}$ for $t \in\left[\tau_{1}, S^{k}\right)$, and, consequently $\phi\left(t, B_{t}, Y_{t}^{k}\right)$ is a local submartingale on $\left[\tau_{1}, S^{k}\right)$.

Since $\mathrm{Cl} \operatorname{dom} \phi \subseteq \operatorname{dom} \tilde{\phi}$ and $\left(t, B_{t}, Y_{t}^{k}\right) \stackrel{\text { ucp }}{\rightarrow}\left(t, B_{t}, Y_{t}\right)$, we necessarily have $\mathbb{P}\left[S_{k} \neq\right.$ $\left.\tau_{2}\right] \rightarrow 0$. Together with the fact that $\left(t, B_{t}, Y_{t}^{k}\right) \stackrel{\text { ucp }}{\rightarrow}\left(t, B_{t}, Y_{t}\right)$, this implies that

$$
\tau_{1}\left(t, B_{t}, Y_{t}^{k}\right)^{S^{k}} \stackrel{\text { ucp }}{\rightarrow} \tau_{1}\left(t, B_{t}, Y_{t}\right)^{\tau_{2}}
$$

Now since $\phi$ is Lipschitz, it follows that

$$
{ }^{\tau_{1}} \phi\left(t, B_{t}, Y_{t}^{k}\right)^{S^{k}} \stackrel{\text { ucp }}{\rightarrow} \tau_{1} \phi\left(t, B_{t}, Y_{t}\right)^{\tau_{2}} .
$$

It remains to use the fact that the class of local submartingales is closed under the ucp convergence. 


\section{$3.2 \quad$ A template for existence}

We turn to another application of our main result Theorem 2.5, namely to the existence of solutions to (2.1) in dimension 1. In order to highlight where the one-dimensional structure is used (and hence what difficulties must be overcome to extend to systems), we start by proving a sufficient condition for existence which holds in any dimension. In the remainder of the paper, we will work with the following standard assumption.

Assumption 3.2 (Growth and regularity of the driver). The driver $f:[0, T] \times$ $\mathbb{R}^{n} \times \mathbb{R}^{n \times d} \rightarrow \mathbb{R}^{n}$ is jointly continuous and there exists a constant $M$ such that

1. (Regularity) for all $t \in[0, T]$ as well as $(y, z),\left(y^{\prime}, z^{\prime}\right) \in \mathbb{R}^{n} \times \mathbb{R}^{n \times d}$, we have

$$
\left|f\left(t, y^{\prime}, z^{\prime}\right)-f(t, y, z)\right| \leq M|y-y|+M\left(1+|y|+\left|y^{\prime}\right|+|z|+\left|z^{\prime}\right|\right)\left|z-z^{\prime}\right|
$$

2. (Growth) for all $t, y, z \in[0, T] \times \mathbb{R}^{n} \times \mathbb{R}^{n \times d}$,

$$
|f(t, y, z)| \leq+M\left(1+|y|+|z|^{2}\right) .
$$

The main idea behind our proof is inspired by the work of Darling [Dar95] in stochastic differential geometry, which is, in turn, based on ideas of Picard [Pic94]. We start in a standard way and build a sequence of approximate equations with Lipschitz coefficients: let $\pi_{R}$ denote the orthogonal projection $\pi_{R}: \mathbb{R}^{d} \rightarrow \mathbb{R}^{d}$ onto the closed ball of radius $R$ centered at the origin. We set

$$
f^{k}=f\left(\cdot, \cdot, \pi_{k}(\cdot)\right),
$$

and note that $f^{k}$ is uniformly Lipschitz in $(y, z)$ and that it satisfies Assumption 3.2 with the same constant $M$ as $f$. Standard theory (see, e.g. [Zha17, Theorem 4.3.1, p. 84]) guarantees existence of a unique solution $\left(Y^{k}, Z^{k}\right)$ to

$$
d Y^{k}=-f^{k}\left(\cdot, Y^{k}, Z^{k}\right) d t+Z^{k} \cdot d B, Y_{T}^{k}=\xi,
$$

with $Z^{k} \in \mathcal{L}^{2}$.

Definition 3.3. We will say that the sequence $\left(Y^{k}, Z^{k}\right)$ is a bounded approximation scheme if $\sup _{k}\left(\left\|Y^{k}\right\|_{\mathcal{S}_{\infty}}+\left\|Z^{k}\right\|_{\text {bmo }}\right)<\infty$. We call the sequence $\left(Y^{k}, Z^{k}\right)$ uniformly integrable in probability if $\mathbb{P}\left[\int_{0}^{T}\left|Z^{k}\right|^{2} 1_{\left|Z^{k}\right|^{2}>1 / k} d t>k\right] \rightarrow 0$ as $k \rightarrow \infty$. 
Definition 3.4. We say that the driver $f$ is ucp stable if the following holds: if $\left(Y^{k}, Z^{k}\right)$ is a sequence of solutions to (2.1) with $\sup _{k}\left\|Z^{k}\right\|_{\text {bmo }}<\infty$, and $\left\{Y_{T}^{k}\right\}_{k \in \mathbb{N}}$ is Cauchy in $\mathbb{L}^{p}$ for all $1 \leq p<\infty$, then $\left\{Y^{k}\right\}_{k \in \mathbb{N}}$ is Cauchy with respect to ucp convergence.

Proposition 3.5 (Template for existence). Suppose that $f$ is ucp stable, satisfies Assumption 3.2, and satisfies the condition (AB) locally. Suppose further that the sequence $\left(Y^{k}, Z^{k}\right)$ constructed above is bounded and uniformly integrable in probability. Then, there exists a solution $(Y, Z) \in \mathcal{S}^{\infty} \times$ bmo to (2.1).

Before proving Proposition 3.5, we use the solutions $\left(Y^{k}, Z^{k}\right)$ defined above to introduce the following sequence of forward SDEs with random coefficients:

$$
X_{0}^{k}=Y_{0}^{k}, d X^{k}=-f^{k}\left(\cdot, X^{k}, Z^{k}\right) d t+\pi_{k}\left(Z^{k}\right) \cdot d B
$$

Standard theory (see, e.g., [Zha17, Theorem 3.3.1, p. 68] and [Zha17, Theorem 3.4 .3 , p. 72]) guarantees that (3.2) has a unique strong solution $X^{k}$ which lies in $\cap_{p \geq 1} \mathcal{S}^{p}$. A key observation here is that, since $\pi_{k}$ is a projection, the pair $\left(X^{k}, \pi_{k}\left(Z^{k}\right)\right)$ satisfies the same equation (3.1) as $\left(Y^{k}, Z^{k}\right)$. Of course, the terminal value $X_{T}^{k}$ of $X^{k}$ will, in general, differ from $Y_{T}^{k}=\xi$. The following Lemma follows from standard stability results for SDEs, together with the assumption that $\left(Y^{k}, Z^{k}\right)$ is bounded and uniformly integrable in probability.

Lemma 3.6. Under the hypotheses of Proposition 3.5, and with $Y^{k}$ and $X^{k}$ defined in (3.1) and (3.2) above, we have

$$
Y^{k}-X^{k} \rightarrow 0 \text { in } \mathcal{S}^{p} \text { for each } p \in[1, \infty)
$$

In particular $X_{T}^{k} \rightarrow \xi$, in $\mathbb{L}^{p}$ for each $p \in[1, \infty)$.

Now we report the proof of Proposition 3.5.

Proof of Proposition 3.5. Lemma 3.6, together with the assumption that $f$ is ucp stable, implies that $\left\{X^{k}\right\}_{k \in \mathbb{N}}$ is Cauchy with respect to ucp convergence. Thus we identify a process $Y$ such that $X^{k} \stackrel{\text { ucp }}{\rightarrow} Y$. Since $X_{T}^{k} \rightarrow \xi$ in $\mathbb{L}^{p}$, it necessarily follows that $Y_{T}=\xi$. Applying Theorem 3.1 shows that $Y$ is a solution to (2.1) with terminal condition $\xi$. Standard estimates then give the desired regularity.

Remark 3.7. There are various conditions which guarantee that the sequence $\left(Y^{k}, Z^{k}\right)$ is bounded, even for systems. For example, if $f$ satisfies the condition (AB), then 
$\left(Y^{k}, Z^{k}\right)$ is bounded - see the proof of Theorem 2.14 in [XŽ18]. However, when $n>1$ we do not currently have simple conditions which guarantee $f$ is ucp stable or that the sequence $\left(Y^{k}, Z^{k}\right)$ is uniformly integrable in probability - this is an issue we hope to address in future research. However, we note that the hypotheses of Proposition 3.5 can be verified when $f$ is the geometric driver appearing in (1.2), at least under certain geometric conditions on the corresponding connection - this is exactly the program carried out in [Dar95]. It is also possible to verify the hypotheses of Proposition 3.5 when $n=1$, again under appropriate technical assumptions. Indeed, when $n=1$ ucp stability can be shown through a change of measure argument (see for example the proof of Proposition 2.3 in [BE13b]), while uniform integrability in probability can be obtained by adapting the coupling argument in [Dar95].

\section{References}

[BE13a] Pauline Barrieu and Nicole El Karoui, Monotone stability of quadratic semimartingales with applications to unbounded general quadratic BSDEs, Ann. Probab. 41 (2013), no. 3B, 1831-1863.

[BE13b] P. Briand and R. Elie, A simple constructive approach to quadratic BSDEs with or without delay, Stochastic Process. Appl. 123 (2013), no. 8, 29212939 .

[Bis73] J.-M. Bismut, Conjugate convex functions in optimal stochastic control, J. Math. Anal. Appl. 44 (1973), 384-404.

[Bla05] Fabrice Blache, Backward Stochastic Differential Equations on Manifolds, Probability Theory and Related Fields 132 (2005).

[Bla06] Fabrice Blache, Backward Stochastic Differential Equations on Manifolds II, Probability Theory and Related Fields 136 (2006), 234-262.

[Car16] René Carmona, Lectures on BSDEs, stochastic control, and stochastic differential games with financial applications (financial mathematics), SIAMSociety for Industrial and Applied Mathematics, 2016.

[CN17] Patrick Cheridito and Kihun Nam, BSE's, BSDE's and fixed-point problems, Ann. Probab. 45 (2017), no. 6A, 3795-3828. 
[Dar95] R.W. Darling, Constructing Gamma-martingale with prescribed limit, using backward SDE, Ann. Probab. 23 (1995), no. 3, 1234-1261.

[Éme89] Michel Émery, Stochastic calculus in manifolds, Universitext, SpringerVerlag, Berlin, 1989, With an appendix by P.-A. Meyer.

[HR19] Jonathan Harter and Adrien Richou, A stability approach for solving multidimensional quadratic BSDEs, Electron. J. Probab. 24 (2019).

[HT16] Ying Hu and Shanjian Tang, Multi-dimensional backward stochastic differential equations of diagonally quadratic generators, Stochastic Process. Appl. 126 (2016), no. 4, 1066-1086.

[JKL17] Asgar Jamneshan, Michael Kupper, and Peng Luo, Multidimensional quadratic BSDEs with separated generators, Electron. Commun. Probab. 22 (2017), Paper No. 58, 10.

[JS03] Jean Jacod and Albert N. Shiryaev, Limit theorems for stochastic processes, second ed., Grundlehren der Mathematischen Wissenschaften [Fundamental Principles of Mathematical Sciences], vol. 288, Springer-Verlag, 2003.

[Ken90] W. S. Kendall, Probability, convexity, and harmonic maps with small image. I. Uniqueness and fine existence, Proc. London Math. Soc. (3) 61 (1990), no. 2, 371-406.

[Ken91] Wilfrid S. Kendall, Convex geometry and nonconfluent $\Gamma$-martingales. I. Tightness and strict convexity, Stochastic analysis (Durham, 1990), London Math. Soc. Lecture Note Ser., vol. 167, Cambridge Univ. Press, Cambridge, 1991, pp. 163-178.

[Ken92] — Convex geometry and nonconfluent $\Gamma$-martingales. II. Wellposedness and $\Gamma$-martingale convergence, Stochastics Stochastics Rep. 38 (1992), no. 3, 135-147.

[Ken94] _ Probability, convexity, and harmonic maps. II. Smoothness via probabilistic gradient inequalities, J. Funct. Anal. 126 (1994), no. 1, 228257. 
[Kob00] M. Kobylanski, Backward stochastic differential equations and partial differential equations with quadratic growth, Ann. Probab. 28 (2000), no. 2, 558-602.

[Nam19] Kihun Nam, Global well-posedness of non-markovian multidimensional superquadratic bsde, ArXiv e-prints 1912.03692, 2019.

[Pha09] Huyên Pham, Continuous-time stochastic control and optimization with financial applications, Stochastic Modelling and Applied Probability, vol. 61, Springer-Verlag, Berlin, 2009.

[Pic94] Jean Picard, Barycentres et martingales sur une variété, Ann. Inst. H. Poincaré Probab. Statist. 30 (1994), no. 4, 647-702.

[PP90] É. Pardoux and S. Peng, Adapted solution of a backward stochastic differential equation, Systems Control Lett. 14 (1990), no. 1, 55-61.

[Tev08] R. Tevzadze, Solvability of backward stochastic differential equations with quadratic growth, Stochastic Process. Appl. 118 (2008), no. 3, 503-515.

[XŽ18] Hao Xing and Gordan Žitković, A class of globally solvable markovian quadratic BSDE systems and applications, Ann. Probab. 46 (2018), no. 1, 491-550.

[Zha17] J. Zhang, Backward Stochastic Differential Equations, from Linear to Fully Nonlinear Theory, Springer, 2017. 